\title{
Fabrication and characterization of a microsystem for a micro-scale heat transfer study
}

\author{
Linan Jiang $\dagger$, Yuelin Wang $\neq$, Man Wong $\dagger$ and Yitshak Zohar $\dagger \S$ \\ $\dagger$ Hong Kong University of Science and Technology, Hong Kong \\ $\$$ Zhejiang University, Hangzhou, China \\ E-mail: mezohar@ust.hk
}

Received 9 August 1999

\begin{abstract}
A micro-system consisting of micro-channels with integrated temperature sensors was successfully designed and fabricated for the study of the heat-transfer properties of fluid flow in micro-domains. Surface micro-machining technology was used to construct the micro-channels with dimensions of about $40 \mu \mathrm{m} \times 1.4 \mu \mathrm{m} \times 4000 \mu \mathrm{m}$. Polysilicon thermistors, $4 \mu \mathrm{m} \times 4 \mu \mathrm{m} \times 0.4 \mu \mathrm{m}$ in size were suspended across the channels and directly exposed to the fluid for local temperature measurements. The micro-channels and the micro-sensors were calibrated and the micro-system was characterized. The integrated micro-system performance was theoretically analysed to provide a framework for the interpretation of the experimental data, and the various heat-transfer mechanisms are subsequently discussed.
\end{abstract}

\section{Introduction}

Today with the trend towards greater miniaturization and higher component density of semiconductor devices, a variety of high-density, high-power, high-speed microelectronic devices are packaged in chips. Thus heat removal and control has become a challenging task and heat-transfer engineering is increasingly playing an important role in this field. Most of the solutions proposed thus far to meet the requirement of the heat removal for VLSI are based on a micro-heat sink [1]. These micro-heat sinks commonly consist of an array of micro-channels. Thus, the heat transfer properties of the micro-channel flow need to be understood for the optimal design of the heat sink. Some investigations on heat transfer in the micro-channel have been carried out. However, most of the results are based on theoretical analyses [2-5], while very little experimental data are available. It is questionable whether currently used heat transfer models are adequate in analysing micro-meter scale problems, since classical theories are based on the continuum assumption which may not be valid for micro-systems [6-10]. Therefore, basic experimental studies on heat transfer in micro-domains are needed to reveal the deviation from a macro-scale field, and further develop a suitable model for micro-scale systems.

The rapid advances in micro-machining technology have made it possible to experimentally investigate heat transfer problems on a micro-scale. Temperature micro-sensors can be integrated into micro-channels on a single chip, providing

$\S$ Corresponding author. Hong Kong University of Science and Technology, Clear Water Bay, Kowloon, Hong Kong. a suitable test vehicle for micro-scale heat transfer studies. The experimental results can then be used to evaluate existing heat transfer models for micro-systems. In this work, a micro-system consisting of micro-channels integrated with temperature micro-sensors has been designed and fabricated to examine the relevant heat-transfer mechanisms. Direct measurements of temperature distribution along the microchannels are presented, for the first time, for a variety of test conditions demonstrating the potential of such thermal micro-systems.

\section{Device design and fabrication}

Heat-transfer studies require sensors to measure the temperature field, which is the most fundamental property. In the current devices, the sensors are designed as thermistors. The working principle of a thermistor is based on the temperature dependence of the electrical resistivity of the materials. The thermistors were designed in a mechanical bridge configuration using doped polysilicon films [11] to satisfy the sensitivity and stability requirements. The two ends of the mechanical bridge, serving as electrical conductors and mechanical supports, were heavily doped for low temperature sensitivity and low electrical resistivity. The 'sensing' region at the centre was lightly doped for optimal parameter control, temperature sensitivity and operational stability to ensure certain resistance and ohmic heating.

The micro-channels, shown in figure 1 , were designed to be either $4 \mathrm{~mm}$ or $5 \mathrm{~mm}$ in length, either $20 \mu \mathrm{m}$ or $40 \mu \mathrm{m}$ in width and $1.4 \mu \mathrm{m}$ in height. The temperature sensors were 


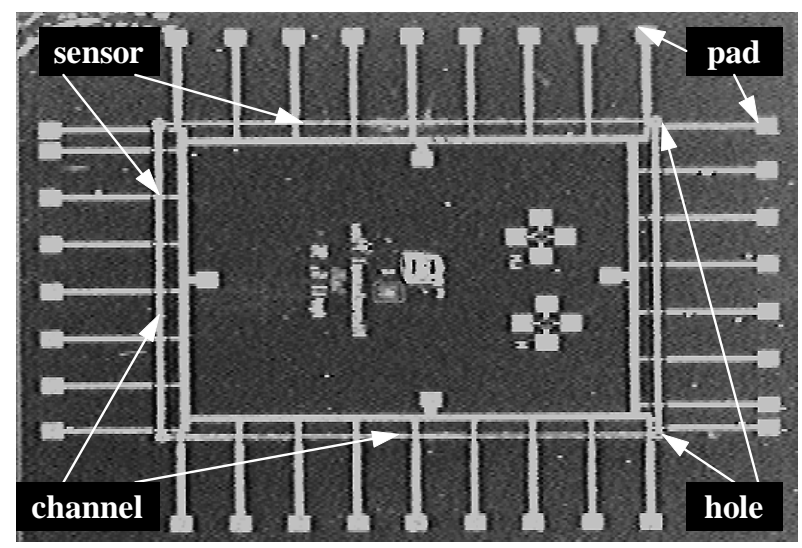

Figure 1. A photograph of the top view of the micro-device.

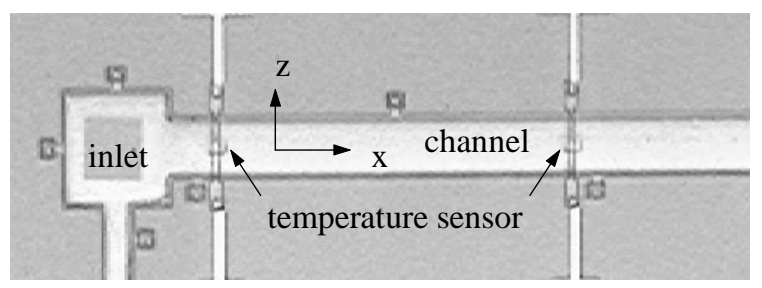

Figure 2. A picture showing a micro-channel with integrated temperature sensors.

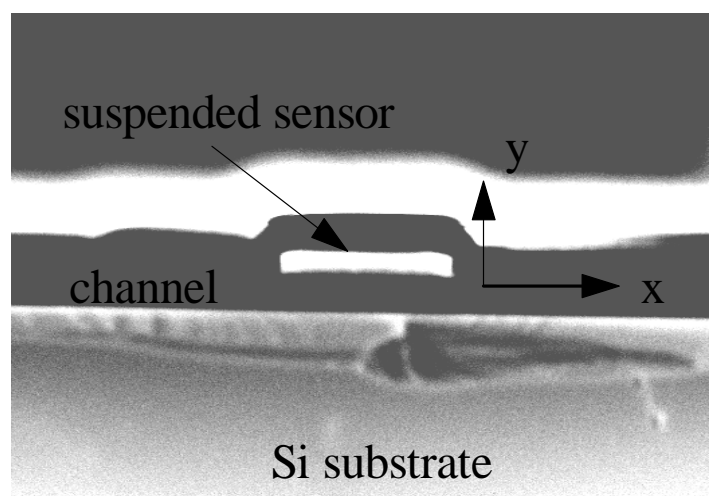

Figure 3. A cross sectional SEM graph showing a temperature sensor suspended in the middle of a micro-channel.

distributed along the length of the channel at either a $300 \mu \mathrm{m}$ or a $600 \mu \mathrm{m}$ interval, as shown in figure 2 . In order to sense the local fluid temperature inside the channel, the thermistors were suspended across the channel directly exposed to the fluid, as shown in figure 3. The sensor thickness was made as small as possible, about $0.4 \mu \mathrm{m}$, in an attempt to minimize the blockage effect in the micro-channel.

Schematic cross sections of the major fabrication steps are shown in figure 4 . The starting substrates were $100 \mathrm{~mm}$ p-type silicon wafers with thickness of $550 \mu \mathrm{m}$ and resistivity of $10-15 \Omega \mathrm{cm}$. The fabrication began with the formation of a stack of $0.5 \mu \mathrm{m}$ thermal oxide underneath a $0.2 \mu \mathrm{m}$ LPCVD $840^{\circ} \mathrm{C}$ low-stress silicon nitride layer (figure 4(a)). Following the definition of the $45 \mu \mathrm{m}$ fluid inlet/outlet holes, drilled from the rear of the wafers using a $35 \mathrm{wt} \%$ aqueous $\mathrm{KOH}$ solution at $85^{\circ} \mathrm{C}$, the nitride layer was removed. The remaining thermal oxide was patterned to form the bulk of the lower half of the channel. A $0.2 \mu \mathrm{m}$ phosphosilicate

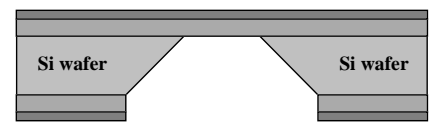

(a)

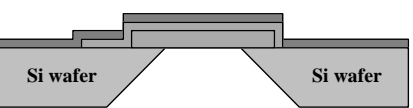

(b)

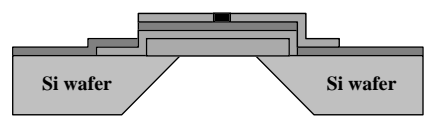

(c)

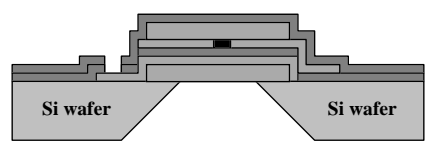

(d)

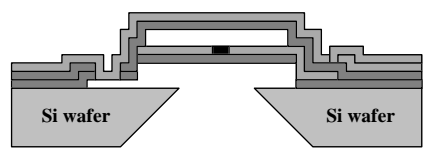

(e)

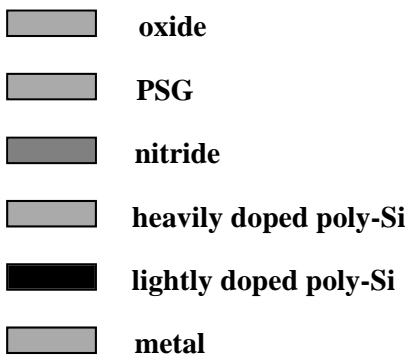

Figure 4. Major fabrication steps of the integrated micro-system.

glass (PSG) layer was deposited and patterned to form the etch channels for the sacrificial layers, which also added $0.2 \mu \mathrm{m}$ to the thickness of the lower half of the channel (figure 4(b)). After the deposition and patterning of $0.2 \mu \mathrm{m}$ silicon nitride for electrical and thermal insulation between the substrates and the sensors, a $0.4 \mu \mathrm{m}$ undoped polysilicon layer was deposited at $620^{\circ} \mathrm{C}$ and patterned in a 'dumbbell' shape. The thermistor was formed by selectively implanting phosphorus into a $4 \mu \mathrm{m}$ stretch of polysilicon in the middle of the narrow portion of the dumbbell (figure 4(c)). The dosage was $5 \times 10^{14} \mathrm{~cm}^{-2}$ and the implant energy was $150 \mathrm{keV}$. The rest of the layer was heavily doped with $5 \times 10^{15} \mathrm{~cm}^{-2}$ arsenic to reduce the interconnect resistance. The nitride diffusion barrier prevented dopant migration from the PSG to the sensor region. The top half of the channel was next defined by a $0.7 \mu \mathrm{m} 400^{\circ} \mathrm{C}$ low temperature oxide (LTO) deposition and patterning. This was followed by a $1 \mu \mathrm{m}$ low stress nitride deposition, which would form the 'frame' of the channel (figure 4(d)). After the removal of the sacrificial layers in a $30 \mathrm{wt} \%$ aqueous HF solution through the etching holes, the sensors were left suspended in the middle of the channel. A $0.5 \mu \mathrm{m}$ LTO layer was deposited to seal the etching holes. Finally, contact windows were opened on the heavily doped polysilicon pads, Al-Si was sputter deposited, patterned, and sintered at $450{ }^{\circ} \mathrm{C}$ for $30 \mathrm{~min}$ (figure $4(\mathrm{e})$ ). 


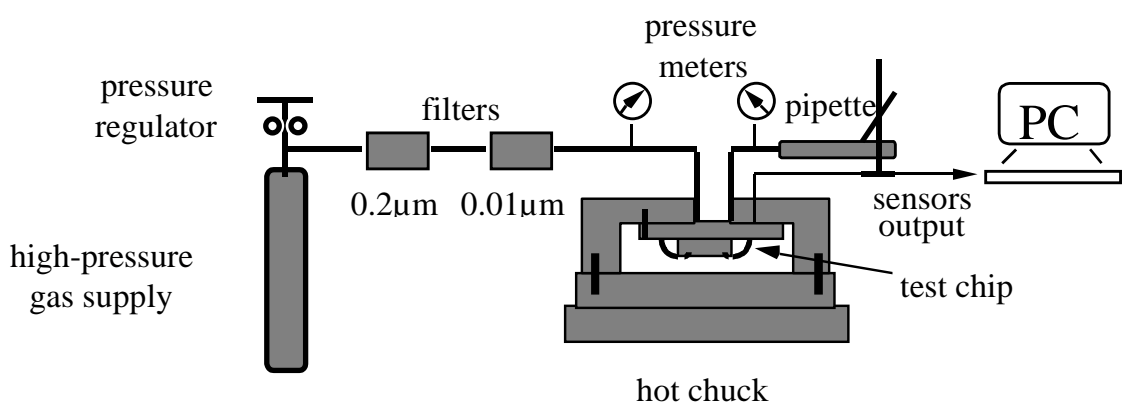

Figure 5. Experimental set-up.

\section{Experimental set-up}

The experimental apparatus, schematically shown in figure 5, consisted of two parts: (i) the external fluid handing system; and (ii) the micro-channel test chip. The external fluid handling system included a pressurized fluid reservoir, a pressure regulator, filters, a pressure transducer, a thermocouple, a hot chuck with a temperature controller, inlet/outlet adapters and a pipette. The micro-channel chip, together with the thermocouple, was mounted on the hot chuck, where the temperature was controlled over a wide range from 0 to $300^{\circ} \mathrm{C}$. Three different gases, nitrogen, helium and argon, were used as the working fluids. The gases were supplied directly from regulated, pressurized cylinders such that the driving pressure could be adjusted. The working gas was passed through a $0.2 \mu \mathrm{m}$ and a $0.01 \mu \mathrm{m}$ filter, before entering the test micro-channel, to prevent channel plugging. Ceramic adapters with a flange were glued to the back of the chip, connecting the external system to the micro-channel and enabling the flow of gases through the entire system without any leakage.

\section{Micro-system calibration}

The micro-system consisted of two main components: the micro-channel flow and the temperature micro-sensors. Both were calibrated to ensure the reliability of the data. The mass flow rate through the micro-channel, $Q_{m}$, was first measured by timing the motion of a meniscus through a precision pipette as a function of the inlet pressure, $p_{i}$, and outlet pressure, $p_{o}$. Figure 6 compares the experimental results with theoretical calculations under different conditions for a micro-channel about $40 \mu \mathrm{m}$ wide, $1.4 \mu \mathrm{m}$ high and $4000 \mu \mathrm{m}$ long. The distinction among the various regimes of the flow can be obtained with the introduction of the Knudsen number $K n$, which is defined as

$$
K n=\frac{\lambda}{L_{s}}
$$

where $\lambda$ is the mean free path and $L_{s}$ is a characteristic flow dimension. The mean free path of the working fluid, nitrogen, is about $0.063 \mu \mathrm{m}$ in the present experiments. The characteristic length scale is taken as the channel hydraulic diameter, about $2.8 \mu \mathrm{m}$. The resulting Knudsen number is about 0.036 and, therefore, the microchannel flow is considered to be in the slip-flow regime. Within this regime, the Navier-Stokes equations may be used with slip boundary

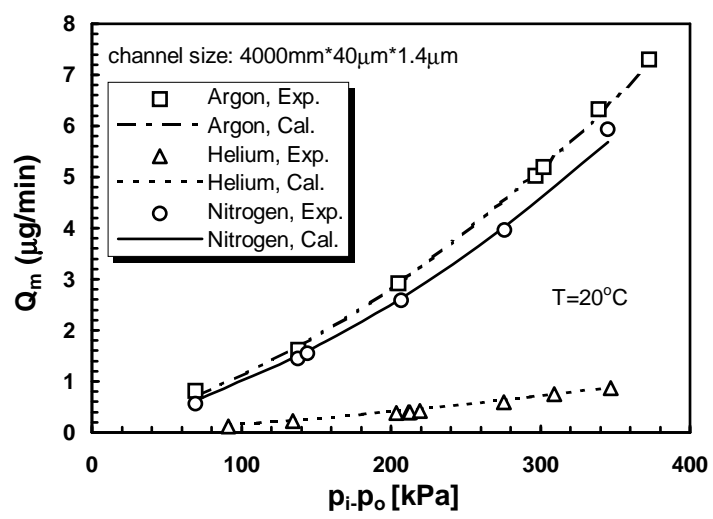

(a)

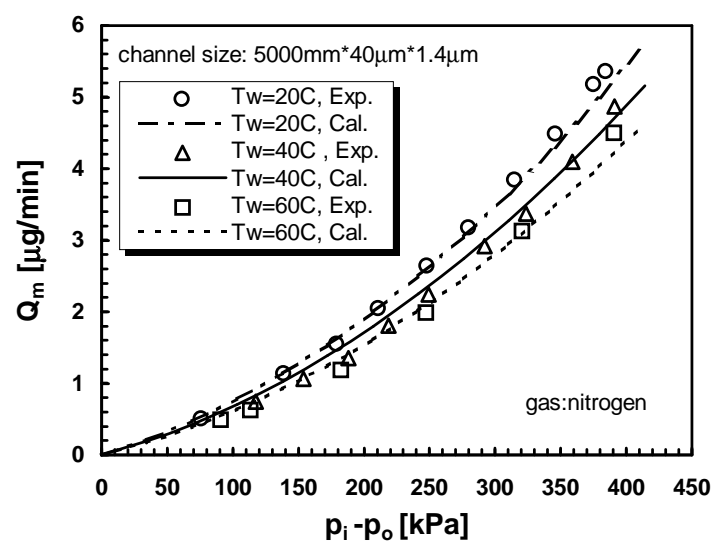

(b)

Figure 6. Mass flow rate variations with the pressure ratio for different: (a) working fluid; and (b) gas temperature.

condition at the wall [12-15]. The corresponding mass flow rate can be calculated as follows

$$
\begin{aligned}
Q_{m} & =\frac{p_{o}{ }^{2} W H^{3}}{24 \mu R T L}\left[\left(\frac{p_{i}}{p_{o}}\right)^{2}-1+12 K n_{o}\left(\frac{p_{i}}{p_{o}}-1\right)\right] \\
& -\frac{Q_{m}^{2}}{4 \pi \mu L} \ln \frac{p_{i}}{p_{o}}
\end{aligned}
$$

where $W, H$ and $L$ are the width, height and length of the channel, respectively. $K n_{o}$ is the outlet Knudsen number, $R$ the specific gas constant, $T$ the gas temperature, $\rho$ the gas density and $\mu$ the gas viscosity. The values of the specific gas constant $R$, and gas viscosity $\mu$, for nitrogen are $0.297 \mathrm{~kJ} \mathrm{~kg}^{-1} \mathrm{~K}^{-1}$ and $1.79 \times 10^{-5} \mathrm{~N} \mathrm{~s} \mathrm{~m}^{-2}$, respectively. The first term in (2) includes compressible effect and slip effect, while the second term includes the gas acceleration effect and non-parabolic velocity profile effect. In this 


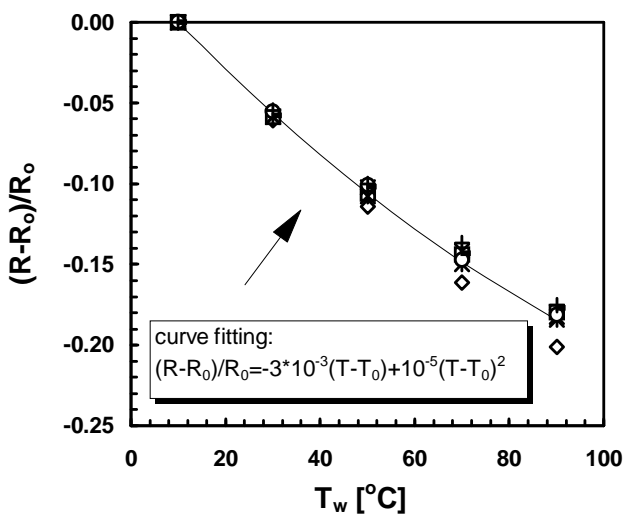

(a)

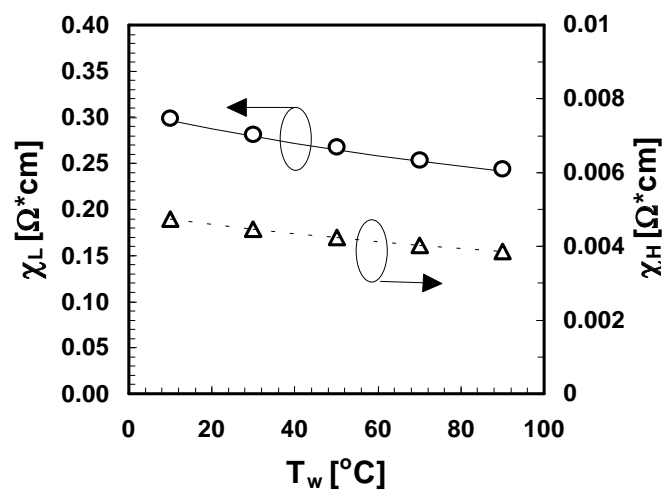

(b)

Figure 7. Calibration of the micro-sensors: (a) resistance dependence of all the sensors; and (b) resistivity dependence of a typical sensor on temperature.

analysis, the pressure losses associated with the channel inlet and outlet are negligible since the length to height ratio is about 2850 [13]. The model has been verified for microchannel flow [16] and, therefore, it was used to calibrate the current micro-channels with the temperature sensors. Figure 6(a) shows the mass flow rate at room temperature as a function of the pressure ratio for three gases: helium, argon and nitrogen. Figure 6(b) shows the mass flow rate for nitrogen at different ambient temperatures: 20, 40 and $60^{\circ} \mathrm{C}$. The resulting curves are clearly nonlinear, mainly due to the compressibility effect. The agreement between the calculations and the measurements is good and, thus, the micro-channels can further be used for heat-transfer studies.

In order to calibrate the temperature micro-sensors, the test chip together with a thermocouple were encapsulated between two aluminium plates, which were mounted on a hot chuck. The chuck was heated up, and its temperature was fixed by a controller. A multi-meter was used to measure the resistance of each sensor $R$, as a function of the temperature recorded by the thermocouple. The results are shown in figure 7(a), where the sensor resistance decreases with increasing temperature, indicating a negative temperature coefficient of resistance (TCR). Furthermore, the curves are nonlinear in the tested temperature range $10-100^{\circ} \mathrm{C}$, and can be fitted by the expression

$$
\frac{R-R_{0}}{R_{0}}=\alpha\left(T-T_{0}\right)+\beta\left(T-T_{0}\right)^{2}
$$

where $R_{0}$ is the sensor resistance at the reference temperature $T_{0}=20^{\circ} \mathrm{C}$. The parameters $\alpha$ and $\beta$ are experimentally found to be about $-3 \times 10^{-3}{ }^{\circ} \mathrm{C}^{-1}$ and $10^{-5}{ }^{\circ} \mathrm{C}^{-2}$, respectively, where $\alpha$ is the linear approximation of the TCR. The calibration of the sensors was conducted with low current $50 \mu \mathrm{A}$, to minimize self-heating. Consequently, the temperature was almost uniform along the thermistor such that the resistivity dependence on temperature of each region can be estimated as follows

$$
R(T)=\frac{\chi_{H}(T) L_{H}+\chi_{L}(T) L_{L}}{a b}
$$

where $\chi_{H}$ and $L_{H}$ are the resistivity and length of the highly-doped region, $\chi_{L}$ and $L_{L}$ are the resistivity and length of the lightly-doped region, and $a$ and $b$ are the sensor width and thickness. The resistance in (4), $R(T)$, can be measured directly leading to one equation with two unknowns. However, the resistivity ratio $\chi_{L} / \chi_{H}$ is estimated to be about 60 due to the given doping schedules in both regions $[17,18]$. Thus, the resulting resistivity dependence on temperature for each region can be calculated and the results are shown in figure $7(\mathrm{~b})$.

\section{Integrated system characterization}

After the calibration of the sensors and the channels, the temperature distribution along the micro-channels was measured with nitrogen serving as the working fluid. The gas inlet temperature was about $20^{\circ} \mathrm{C}$, while the wall temperature was fixed by the controller at 20,50 or $80^{\circ} \mathrm{C}$. The driving pressure varied from $138 \mathrm{kPa}$ to $276 \mathrm{kPa}$ to obtain different mass flow rates for the current testing conditions. The measurements are summarized in figure 8, where the temperature along the channel is almost uniform, and within $1{ }^{\circ} \mathrm{C}$ lower than the wall temperature for all tested conditions.

An accurate theoretical analysis of the system thermal performance, which includes all the slip-flow effects, is complicated. However, a simplified model, which does not account for the temperature jump boundary condition, can still provide reasonable quantitative analysis of the microsystem performance. The steady-state energy balance for the sensor is given by the following equation

$$
\begin{aligned}
& a b k \frac{\mathrm{d}^{2} T}{\mathrm{~d} z^{2}}-2 h(a+b)\left(T-T_{g}\right)-8 \sigma(a+b) T^{3}\left(T-T_{g}\right) \\
& \quad+J^{2} \chi_{0}\left[1+\alpha\left(T-T_{g}\right)\right]=0
\end{aligned}
$$

where $k$ and $\sigma$ are the sensor thermal conductivity and StefanBoltzman constant, respectively. $h$ is the heat-transfer coefficient, $\chi_{0}$ is the sensor resistivity at room temperature, $J$ is the current density through the sensor, $\alpha$ is the TCR and $T_{g}$ is the temperature of the fluid engulfing the sensor. This equation states that the power generated by the current is either stored as thermal energy within the sensor or transferred through heat conduction, convection and radiation to the surrounding. The temperature distribution calculated based on (5), subject to proper boundary conditions for the present sensor configuration [8], is shown in figure 9(a) for different current levels. The calculations are carried out assuming free convection, i.e. no flow inside the channel, and the wall temperature is fixed and equal to the ambient 


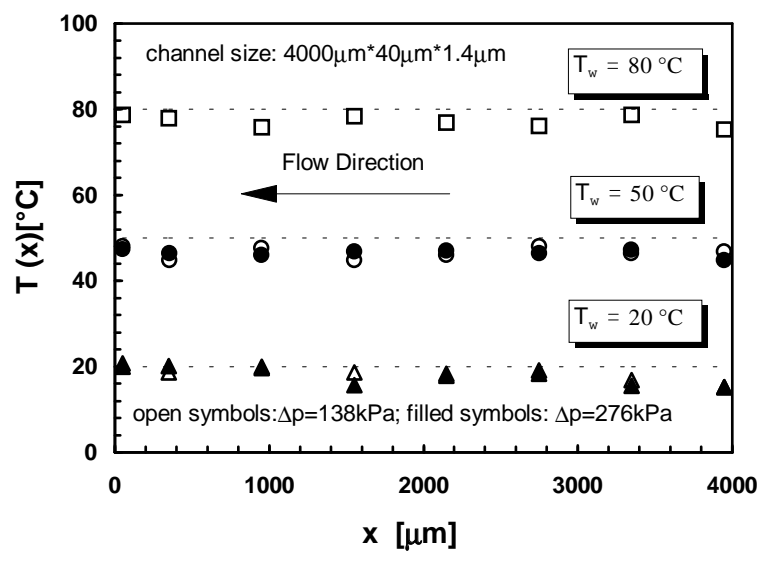

Figure 8. Measured temperature distributions along the micro-channel.

temperature. The distributions are used, together with the calibration curves in figure 7(b), to obtain the resistivity distributions over the sensor and its leads, $\chi(z)$, as depicted in figure 9(b). The total resistance can now be estimated as follows,

$$
R=\int_{-L / 2}^{L / 2} \frac{\chi[T(z)]}{a b} \mathrm{~d} z
$$

where $L$ is the length of the sensor and its leads. The results are compared in figure 10 with direct $I-V$ curve measurements obtained by a Tektronix 370A Programmable Curve Tracer. The agreement between the directly measured and analytically estimated values is satisfactory, so that the model can be used to analyse the energy balance over the sensor.

The experimental results presented in figure 8 were collected under forced convection conditions. Since the sensors were suspended inside the channel, the local bulk temperature of the gas should be taken into account in analysing the output signals of the sensors. A precise analysis of the microsystem under these conditions is a major challenge. However, an approximate approach is presented to highlight the important heat-transfer mechanisms involved. The fluid temperature distribution along the channel is governed by

$$
Q_{m} c_{p} \frac{\mathrm{d} T_{g}}{\mathrm{~d} x}=h L_{p}\left(T_{w}-T_{g}\right)
$$

where $T_{g}$ is the fluid bulk temperature, $T_{w}$ is the wall temperature, $c_{p}$ is the fluid specific heat and $L_{p}$ is the perimeter of the channel cross section. The heat-transfer coefficient $h$, is related to the Nusselt number $N u$, as follows

$$
N u=\frac{h D_{h}}{k_{g}}
$$

where $D_{h}$ is the channel hydraulic diameter and $k_{g}$ is the gas thermal conductivity. Since the Prandtl number is of the order of one for nitrogen, the thermal entry length is about the same as the hydrodynamic entry length, less than $1 \mu \mathrm{m}$ [19], and has been neglected. The solution of (7) with uniform, constant wall-temperature boundary condition is

$$
\frac{T_{g}(x)-T_{0}}{T_{w}-T_{0}}=1-\mathrm{e}^{-x / L_{e}}
$$

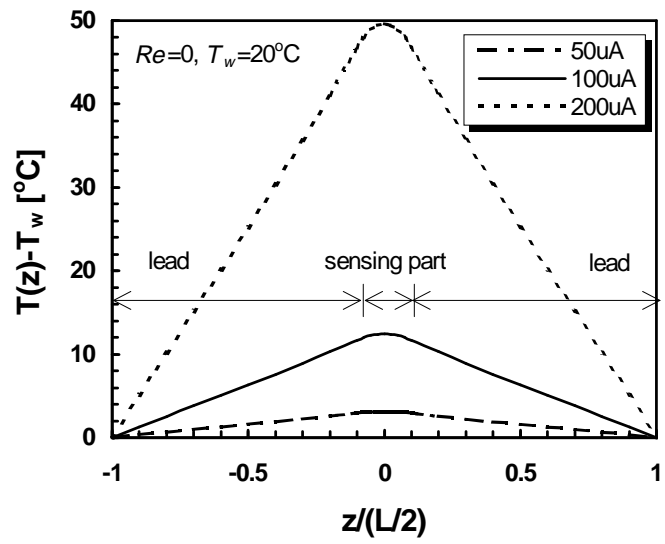

(a)

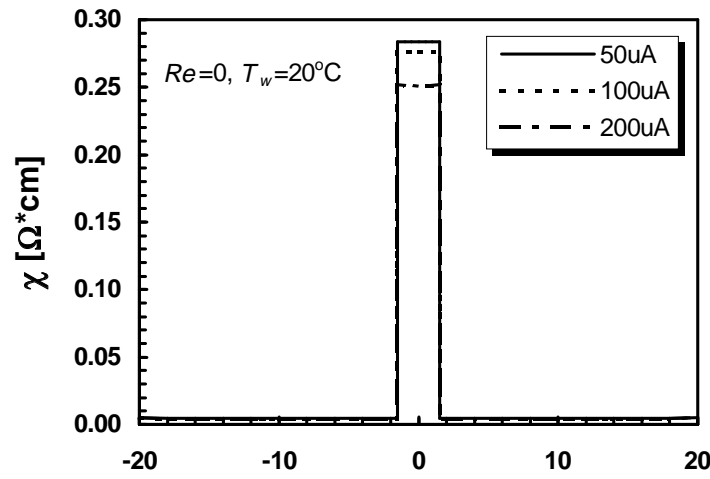

(b)

Figure 9. Effect of current level on the distribution along the sensor of: (a) calculated temperature; and (b) estimated resistivity.

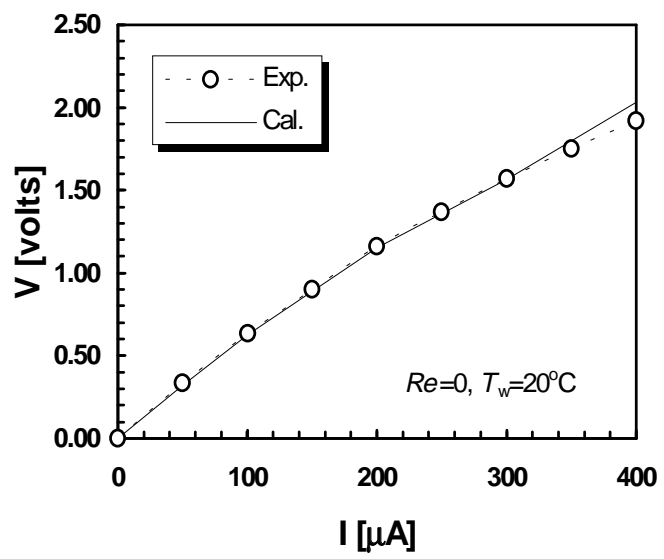

Figure 10. A comparison between measured and calculated $I-V$ characteristics.

where the bulk fluid temperature rises exponentially towards the asymptotic wall temperature. The length scale of the exponential rise $L_{e}$ is defined as

$$
L_{e}=Q_{m} c_{p} /\left(h L_{p}\right)
$$

The Reynolds number for the channel flow is very small, less than 0.2, for which $N u>1$ [19]. The resulting exponential scale is very small, about $0.2 \mu \mathrm{m}$ for $N u=1$, indicating that the bulk temperature of the fluid approaches the wall temperature within a short distance from the inlet. Thus, the fluid bulk temperature is about equal to the wall temperature along the entire length of the micro-channel, and for the 


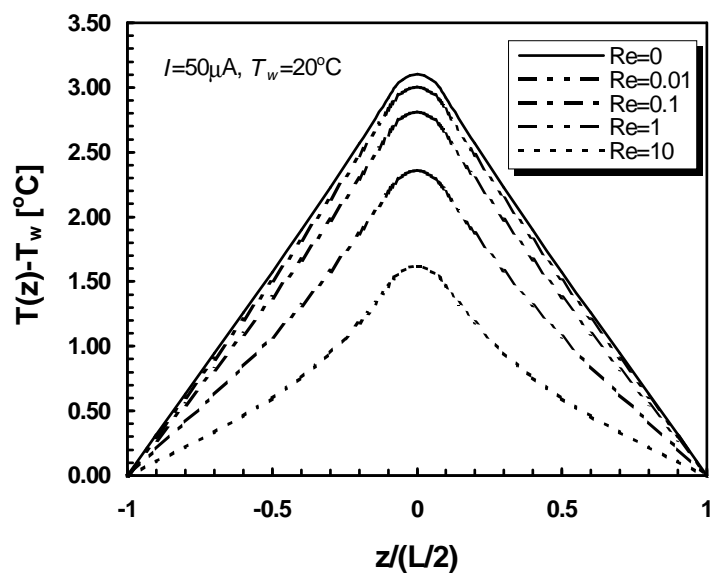

(a)

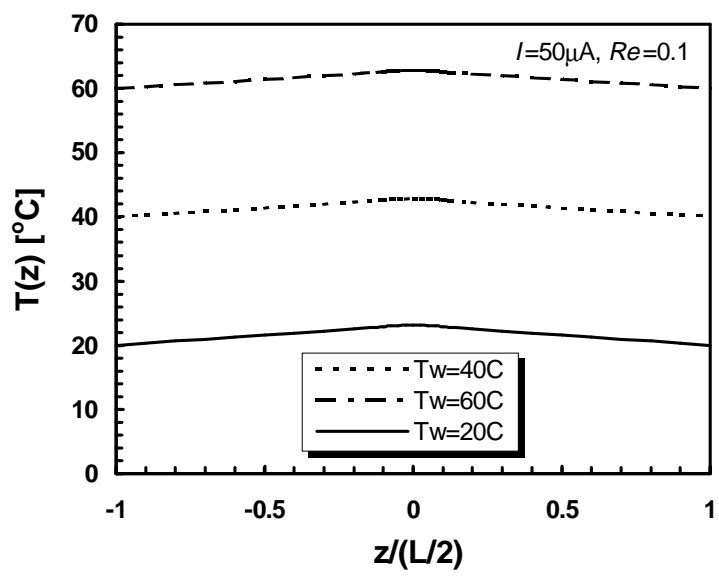

(b)

Figure 11. Calculated temperature distributions along the sensor for different: (a) Reynolds number; and (b) wall temperature.

forced convection analysis of (5), it is reasonable to assume that $T_{g}(x)=T_{g}=T_{w}$.

The effects of Reynolds number $R e$ and wall temperature $T_{w}$ on the temperature distribution along the sensor are summarized in figures 11(a) and 11(b), respectively. All the distributions peak at the middle of the sensor, where the resistivity and subsequently the power dissipation are high. For a given current and wall temperature, shown in figure 11(a), the sensing-element temperature drops markedly with increasing $R e$ because of the enhanced heat convection by the fluid flow. For a given current and Reynolds number, shown in figure 11(b), the sensor temperature increases with the wall temperature. However, the temperature difference between the sensing part and the leads decreases, mainly due to the enhanced heat conduction from the wall to the sensor through the leads.

The calculated dependence of the sensor temperature on the operating conditions is compared in figure 12 with experimental measurements. The combined effect of the wall temperature and the flow rate on the sensing-element temperature is considered for the current of $50 \mu \mathrm{A}$ applied in the present experiments. The agreement between the measured and calculated temperature values is reasonable and, thus, the theoretical model can be used to further analyse the response of the integrated thermal micro-system. Clearly, the sensor temperature drops with increasing Reynolds number due to enhanced convection. However, the relative change in the

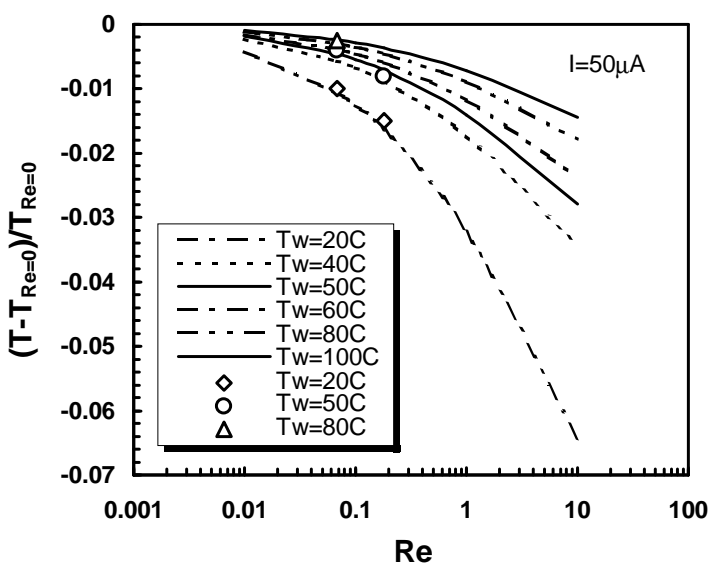

Figure 12. A comparison between calculated and measured temperature dependence on the operating conditions.

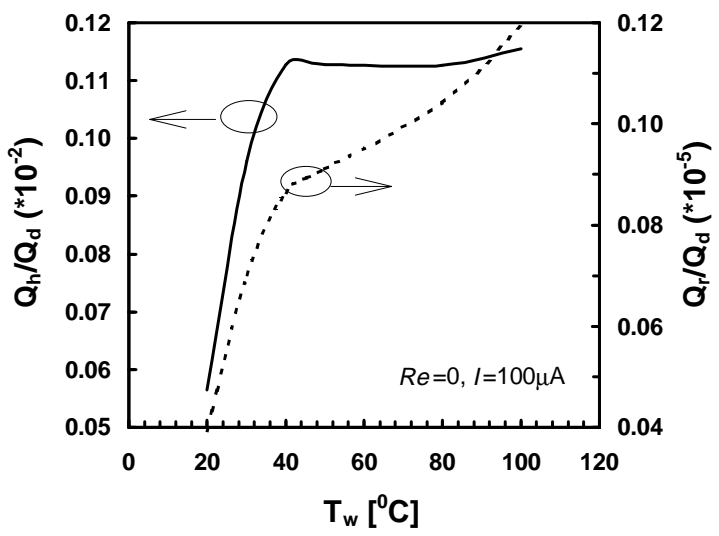

(a)

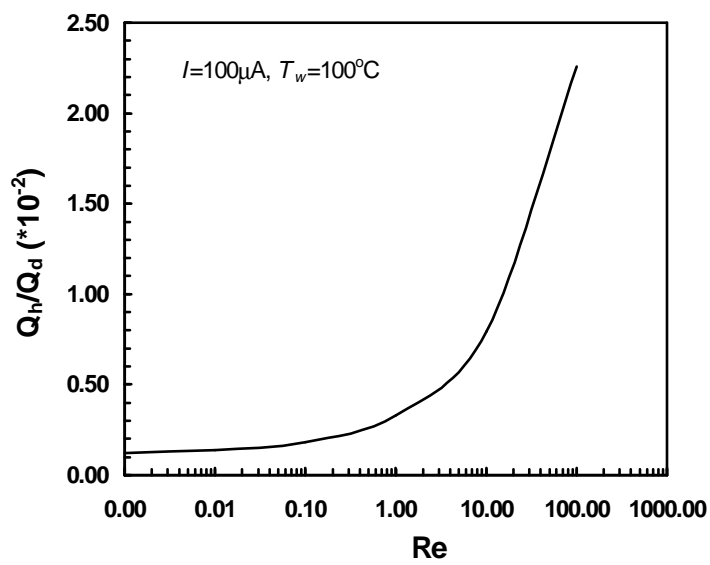

(b)

Figure 13. (a) The relative contribution of radiation, convection and conduction heat transfer from the sensor to the surrounding. (b) The effect of Reynolds number on the ratio between convection and conduction heat loss.

sensor temperature decreases with increasing wall temperature since the fluid flow temperature increases with the wall temperature, and as a result the heat convection decreases.

In order to understand the thermal performance of the micro-system, the relative contribution of the different heat transfer mechanisms involved can be identified. The calculated rates of the heat transfer from the sensor to the surrounding by conduction $Q_{d}$, convection $Q_{h}$, and radiation $Q_{r}$ are presented in figure 13 for a wall temperature of less than $100^{\circ} \mathrm{C}$. The heat loss due to convection is less than 
$0.12 \%$ of the heat loss due to conduction, while the loss due to radiation is of the order of $10^{-6}$ of that due to conduction, as shown in figure 13(a). Hence, the radiation heattransfer mechanism can be ignored altogether. Furthermore, the relative contribution of the convection mechanism is a strong function of the Reynolds number, as demonstrated in figure 13(b). The ratio between convection and conduction heat transfer increases very slowly for $R e<1$, but very quickly for $R e>1$. For all tested operating conditions $R e<$ 0.2 , the ratio is less than $0.2 \%$. Therefore, heat conduction between the wall and the sensing elements through the leads is by far the dominant heat-transfer mechanism in the present thermal micro-system.

\section{Conclusions}

A thermal micro-system consisting of micro-channels with integrated temperature micro-sensors has been successfully fabricated and utilized to provide, for the first time, temperature distributions along a micro-channel. A theoretical model has been applied to analyse the microsystem response to variations in flow rate, driving current and wall temperature. The channel flow temperature approaches the wall temperature within a short distance from the channel inlet and, thus, reduces the effect of forced convection. The radiation heat transfer in the microsystem can be neglected for the present operating conditions. Thus, conduction through the sensor leads is the dominant heat-transfer mechanism governing the sensor temperature. This seems to be unavoidable in all similar systems where temperature sensors are physically connected to metal lines for input/output signals.

\section{Acknowledgment}

The work was sponsored by the Hong Kong Research Grants Council through RGC grant HKUST579/94E.

\section{References}

[1] Tuckerman D B and Pease R F W 1981 IEEE Electron Dev. Lett. 2126

[2] Koh J C Y and Colony R 1986 Int. Commun. Heat Mass Transfer 13 89-98

[3] Weisberg A, Bau H H and Zemel J N 1992 Int. J. Heat Mass Transfer 352465

[4] Mavriplis C, Ahn J C and Goulard K J 1997 J. Thermophys. Heat Transfer 11 489-96

[5] Kavehpour H P and Faghri M 1997 Num. Heat Transfer 32 677-96

[6] Pfahler J, Harley J, Bau H H and Zemel J N 1990 Sensor Actuators A 21-3 431-4

[7] Pfahler J, Harley J, Bau H H and Zemel J N 1991 Micromech. Sensors, Actuators Syst. ASME 49-60

[8] Liu J, Tai Y C, Pong K C and Ho C M 1993 The 7th Int. Conf. on Solid-State Sensors and Actuators p 995

[9] Beskok A and Karniadakis G E 1994 J. Thermophys. Heat Transfer 8 647-55

[10] Harley J C, Huang Y, Bau H H and Zemel J N 1995 J. Fluid Mech. 284 257-74

[11] Jiang L, Wong M and Zohar Y 1999 Meas. Sci. Technol. 10 653-64

[12] Berg H R, Seldam C A and Gulik P S 1993 J. Fluid Mech. $2461-20$

[13] Arkilic E B, Breuer K S and Schmidt M A 1994 Appl. Microfabr. Fluid Mech. ASME 19757

[14] Beskok A, Karniadakis G E and Trimmer W 1996 J. Fluids Eng. 118 448-56

[15] Arkilic E, Schmidt M A and Breuer K S 1997 J. Microelectromech. Syst. 6 167-78

[16] Wu S, Mai J, Zohar Y, Tai Y C and Ho C M 1998 Proc. 11th Ann. Int. Workshop on Micro Electro Mechanical Systems pp 87-92

[17] Jiang L, Wang Y, Wong M and Zohar Y 1999 Proc. 3rd ASME/JSME Joint Fluids Engineering Conf. (San Francisco) FEDSM99-7354

[18] Jiang L, Wang Y, Wong M and Zohar Y 1999 International Mechanical Engineering Congress and Exposition (Nashville) to be presented

[19] Mills A F Heat Transfer (Upper Saddle River, NJ: Prentice-Hall) 